\title{
Occlusive Shrinkage of Ovation Endograft Presenting as Acute Lower Limb Ischemia Effective Endovascular Management
}

\author{
Paolo Bianchi, MD'1, Filippo Scalise, MD, FACC, FESC2, Andrea Mortara, MD, \\ Guido Lanzillo, MD, FECTS ${ }^{4}$, Giuseppe Scardina, $\mathrm{MD}^{3}$, Santi Trimarchi, MD, PhD ${ }^{5}$, \\ Gianfranco Parati, MD, FESC ${ }^{6,7}$, Valerio Tolva, MD, PhD, FEBVS $1,6^{*}$ \\ ${ }^{1}$ Department of Vascular Surgery, Centro Cuore, Policlinico di Monza, Monza, Italy \\ ${ }^{2}$ Department of Interventional Cardiology, Centro Cuore, Policlinico di Monza, Monza, Italy \\ ${ }^{3}$ Department of Cardiology, Centro Cuore, Policlinico di Monza, Monza, Italy \\ ${ }^{4}$ Department of Cardiac Surgery, Centro Cuore, Policlinico di Monza, Monza, Italy \\ ${ }^{5}$ Department of Vascular Surgery, Policlinico San Donato IRCCS, San Donato Milanese, Italy \\ ${ }^{6}$ Department of Health Sciences, University of Milano-Bicocca, Milan, Italy \\ ${ }^{7}$ Istituto Auxologico Italiano IRCCS, San Luca Hospital, Milano, Italy
}

\begin{abstract}
The aim of this report is to describe the imaging and successful treatment of an acute shrinkage of the Ovation Abdominal Stent Graft System. The Ovation Prime system utilizes a polymer-filled sealing ring that is cast in situ at the margin of the aneurysm; however, the residual endograft inner volume after ring filling may reduce volume and graft flow. Nevertheless, there are no reports about severe complications using the Ovation Prime system. A 75-year-old male presented to our hospital for acute lower limb ischemia. The patient reported a previous endograft for abdominal aortic aneurysm 1 month previously, which utilized the Ovation device. Computed tomography (CT) angiography demonstrated a critical narrowing of the endograft at the site of the proximal sealing rings. We decided on urgent treatment, delivering a covered stent graft (CP STENT NUMED ${ }^{T M}$ ). Intraoperative intravascular ultrasound showed effective compaction of the proximal rings. Nine-month follow-up with CT angiography demonstrated good patency without ring recoil of the endograft. This is the first report of endovascular treatment for an acute and symptomatic
\end{abstract}

Published by Science International Corp. ISSN 2325-4637

Fax +12037853552

E-Mail: aorta@scienceinternational.org

http://aorta.scienceinternational.org

shrinkage of proximal rings in the Ovation trivascular endograft. Angiographic and intravascular ultrasound findings showed that covered stenting is effective and that the ring polymer is safely moldable.

Copyright @ 2017 Science International Corp.

Key Words:

Endovascular complication • EVAR • Aortic sealing

\section{Introduction}

Endovascular technology has significantly changed the field of vascular surgery [1-3]. However, approximately one-third of patients presenting with abdominal aortic aneurysm (AAA) are deemed ineligible for standard endovascular aortic repair (EVAR) because of anatomic constraints [1, 2], the majority of which involve inadequacy of the proximal aneurysmal neck. To cope with these challenges, different endovascular approaches have been developed that either enhance stent graft fixation at the proximal

* Corresponding Author:

Valerio Stefano Tolva, MD, PhD, FEBVS

Vascular Surgery Department

Centro Cuore

Policlinico di Monza Via Amati 111, 20900, Monza, Italy

Tel.: +1 39039281 0233; Fax: +1 39039281 0470; E-Mail: valerio.tolva@policlinicodimonza.it 
neck or extend the proximal landing zone to allow adequate apposition to the aortic wall and thus aneurysm exclusion. Patients with aortic necks $<10 \mathrm{~mm}$ have a 4-fold greater risk of proximal endoleak in the first 30 days after operation compared with those with necks $>15 \mathrm{~mm}[1,2]$. Of the available endografts, the Ovation Abdominal Stent Graft System (TriVascular Inc., Santa Rosa, CA, USA) is designed to overcome the limitations of currently available stent grafts. The Ovation graft is suitable for a broad range of aortoiliac characteristics and provides effective sealing for complex proximal infrarenal aortic neck morphology $[4,5]$. However, some authors have observed asymptomatic inflow stenosis at the O-rings of the Ovation stent graft $[6,7]$. Here, we describe the imaging and treatment of an acute complication in a patient treated for AAA with an Ovation stent graft, leading to acute limb ischemia.

\section{Case Presentation}

A 75-year-old man presented to our hospital for acute lower limb ischemia. The patient was referred to our hospital with leg pain at rest as well as foot numbness and hypoesthesia. One month previously, he was treated in another hospital for a rapidly enlarging AAA (from 40 to $48 \mathrm{~mm}$ in 6 months) using an Ovation Abdominal Stent Graft System. His comorbidities were mild chronic obstructive pulmonary disease, obesity (body mass index, 29.3), and hypertension. His symptoms occurred a few hours before he arrived at our emergency room. He complained of severe ankle and calf pain at rest that increased over a 2-hour time span. His femoral arteries were pulseless, and distal bilateral acrocyanosis was present. Blood samples showed increased creatine phosphokinase $(4500 \mathrm{U} / \mathrm{L})$. Critical post-stenotic flowmetric findings were evident upon ultrasound scanning of the femoral arteries. Ankle-brachial pressure index was 0.22 bilaterally. Urgent computed tomography (CT) angiography was performed, which showed severe narrowing of the proximal aortic neck where the endograft rings sealed the AAA (Figure 1A).

Based on these clinical and imaging findings, we decided to perform urgent revascularization. We used an endovascular approach with a
14-F mounted covered stent with high radial force characteristics (CP Stent, NuMED Inc., Hopkinton, NY, USA) with a total covered length of $45 \mathrm{~mm}$. The aortic neck measured $20 \mathrm{~mm}$, and the Ovation stent graft was $29 \mathrm{~mm}$ (Figure 1B). We observed circumferential calcifications surrounding the proximal aortic neck. Under general anesthesia, a 4-F introducer was inserted through the left brachial artery, and an angiogram performed. Intraoperative anticoagulation was achieved using 100 units/kg heparin, with activating clotting time maintained for more than $250 \mathrm{~s}$. The right femoral artery (which was previously used for EVAR) was surgically exposed. An artery pouch was prepared with a $5 / 0$ polypropylene stitch to close the arteriotomy at the end of the procedure. We recorded a clinically significant cycle-averaged pressure drop along the inflow stenosis and further in the endograft main body (45.5 $\pm 15 \mathrm{mmHg}$ ). Mean arterial pressure dropped from 110 to $45 \mathrm{mmHg}$ when measured above and below the stenosis. A Visions PV .035 Digital Intravascular Ultrasound (IVUS) Catheter was placed to calculate the residual lumen and area close to the ring $\left(39.3 \pm 3.5 \mathrm{~mm}^{2}\right)$ and to visualize the echogenicity of the polymer compared with the aortic thrombus (Figure 2). The median area above and below the stenosis was $409 \mathrm{~mm} 2$. CP stent deployment was performed in a two-stage sequence. Final delivery was obtained with high-pressure second stage ballooning (10 ATM) followed by further post-dilation with a Z-MED II ${ }^{\text {TM }}$ Balloon (NuMED Inc., Hopkinton, NY, USA) (Figure 3A). Post-implantation IVUS showed good results in terms of restored area $\left(439 \pm 22 \mathrm{~mm}^{2}\right)$ and reduced ring polymer volume (Figure 3B). The pressure gradient along the endograft neck was eliminated. The patient recovered from limb ischemia and was discharged 5 days after the operation with good bilateral femoral pulses. Nine months later (Figure 3C and 3D), CT angiography showed good patency of the endograft with no neck recoil. Outpatient ultrasound evaluation showed normal flowmetric patterns.

\section{Discussion}

The management of juxtarenal aortic aneurysms with EVAR remains controversial due to the high risks in- 


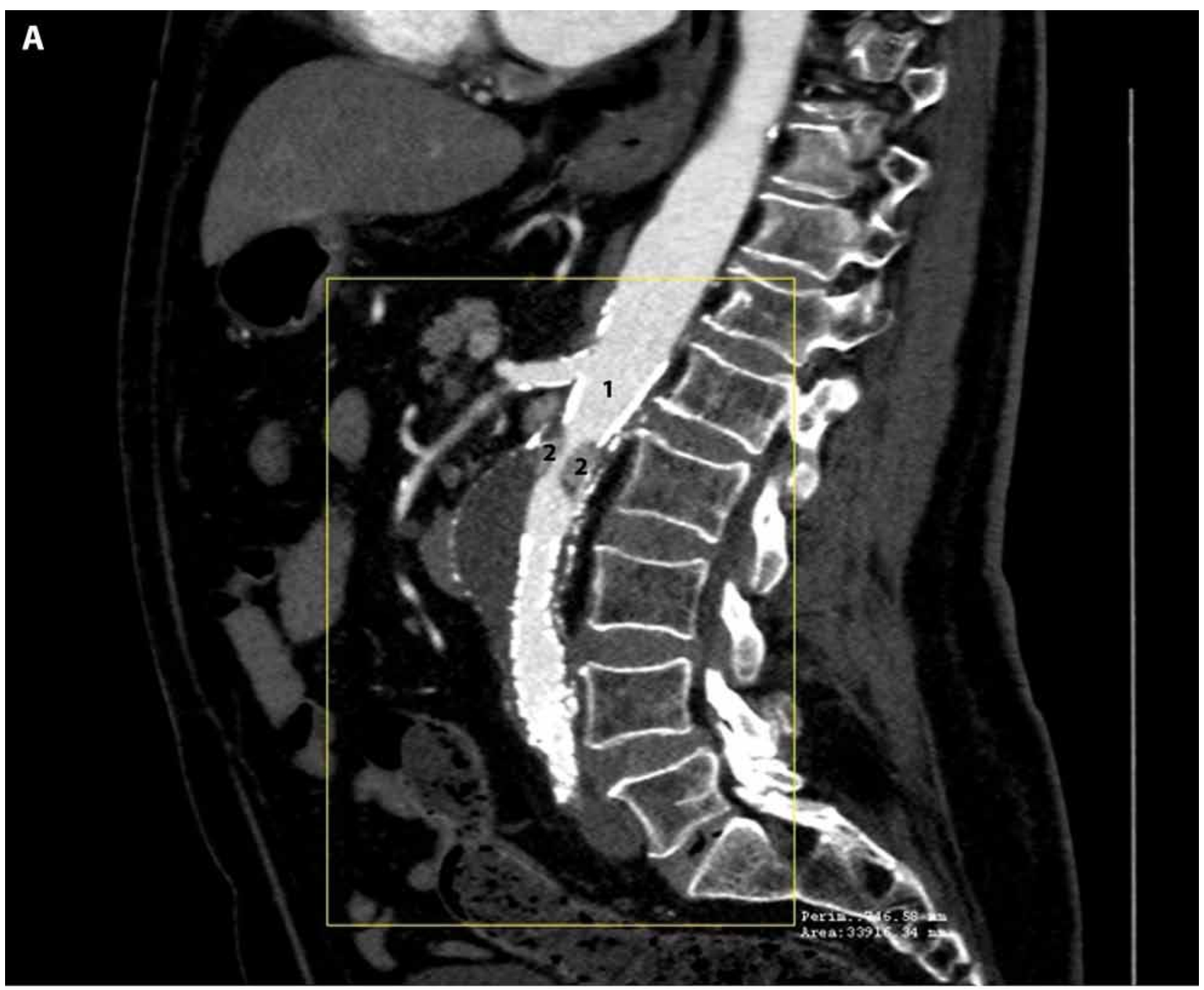

B

stent graft proximal diameter: $29 \mathrm{~mm}$

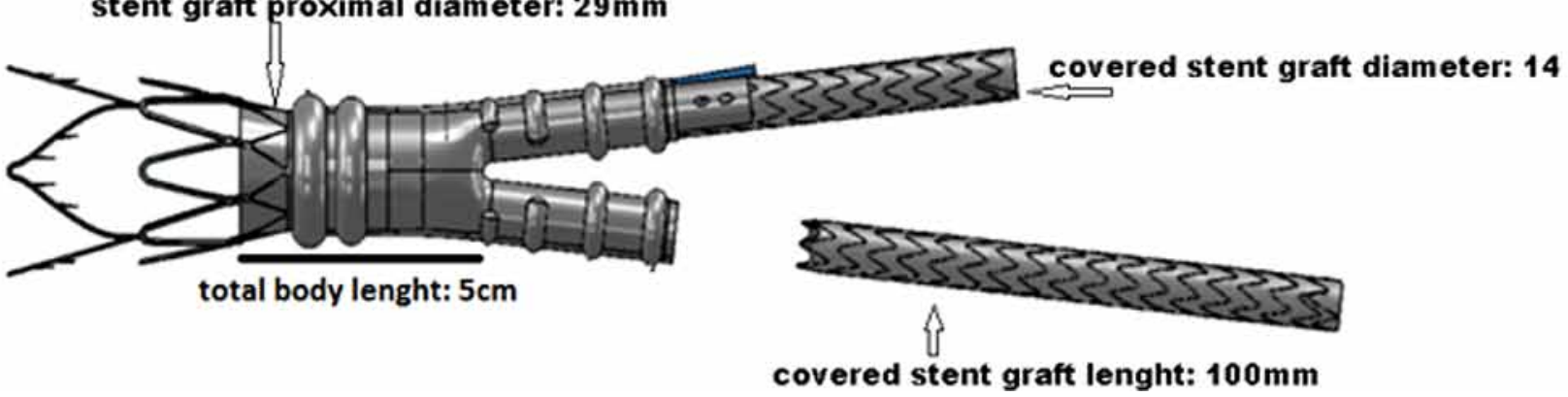

Figure 1. Panel A. Computed tomography angiography showing narrowing of the proximal neck (1 - Bare metal stent of the endograft. 2 - Rings filled with polymer). Panel B. Endograft dimensions.

volved. Therefore, several endovascular techniques have been proposed to ensure a secure proximal landing zone [1-3]. The morphology of the aneurysm neck is especially important in this process [1, 3]. New technol- ogies have expanded the applicability of endovascular aneurysm treatment to cases with anatomically adverse conditions. However, as the indications for mini-invasive techniques have expanded, complications and techni- 


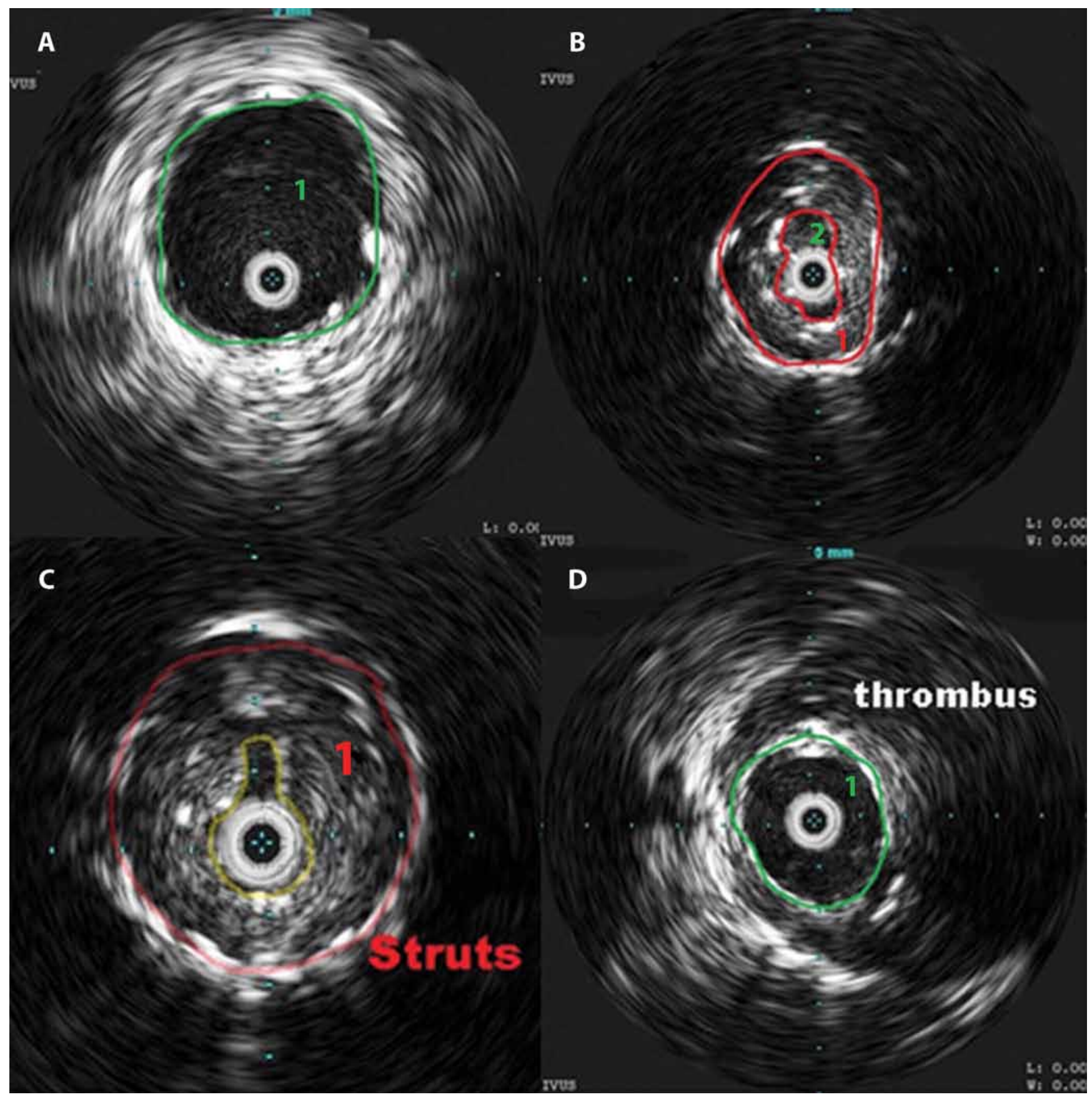

Figure 2. Intravascular ultrasound scanning of the proximal neck. Panel A1. True lumen of the upper part of the endograft just above the ring. Panel B1. Superior ring filled with polymer. Panel B2: Endograft residual lumen. Panel C1 Inferior ring filled with polymer almost occluding the lumen, and the 6-F intravascular ultrasound occupying the residual lumen. Panel D1. True lumen of the lower part of the neck below the inferior ring. The aneurysmal thrombus showed similar echogenicity as the polymer.

cal failure rates have also increased $[4,5,8]$.

In our case, we observed a complication originating from the technical core of the endograft. The Ovation stent graft was conceived to accommodate a broader range of aorto-iliac anatomy with a proximal aortic neck seal mechanism designed to conform to and accommodate the aortic neck $[4,5]$. The aortic body contains a network of inflatable channels that fill dedicated rings 

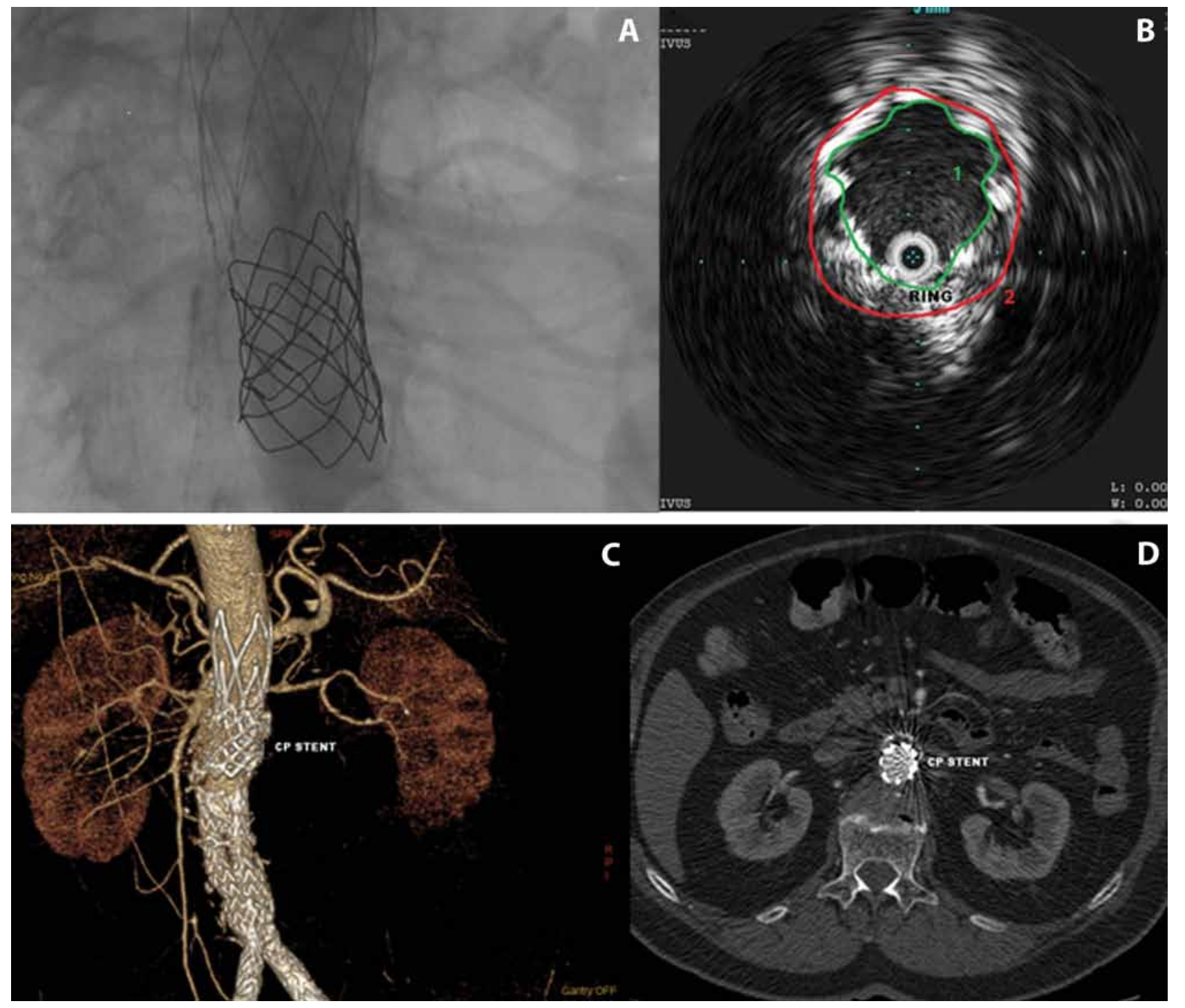

Figure 3. Final result and 9-month computed tomography angiography. Panel A. Angiography results after high-pressure ballooning (10-15 ATM). Panel B. Intravascular ultrasound showing the (Panel A) inner endograft lumen and (Panel B) proximal ring. Panel C and Panel D. Nine-month follow-up showing good patency and sealing of the device.

during deployment with a low-viscosity, radiopaque fill polymer that remains in situ to create an effective seal to the aortic neck. Therefore, aortic neck size and circumferential calcification must be critically evaluated before choosing this endograft. Moderate stenosis has been previously described as a normal finding after Ovation stent graft implantation [6], but the present report appears to be the first to describe a critical narrowing of the aortic neck causing lower limb ischemia.

The sealing mechanism occurs via radial force expressed by the polymer-filled rings. This aortic volume expands externally even though a residual volume occupies the endograft lumen $[6,7]$. If the neck is heavily and circumferentially calcified, the rings may fail to expand outside and converge toward the center of the endograft, narrowing the aortic lumen. Moreover, in our case, ring oversizing (typically about $20 \%$ of the anchoring neck) appeared to be excessive If we consider an original aortic neck of $20 \mathrm{~mm}$, the proper oversizing for the endograft would have been $23 \mathrm{~mm}$. In our patient, the proximal neck of the implanted endograft measured $29 \mathrm{~mm}$. Considering these factors, we speculate that the 
narrowing had two causes: a calcified neck that pushed the rings inward and an excessive ring oversizing that almost completely filled the device lumen.

Mehta et al. reported that $2 \%$ of patients showed aortic body stenosis without re-intervention at 1-year follow-up [6]. Other authors who observed 49 consecutive patients ( 48 men; mean age, $71.2 \pm 7.7$ years) successfully treated with the Ovation abdominal aortic stent graft at a single center concluded that its unique sealing mechanism comes at the expense of a median area inflow stenosis of $\sim 60 \%$ [6]. Moreover, some authors suggest that the coexistence of stenotic wall regions exposed to a high shear rate and post-stenotic recirculation zones may lead to platelet activation and a predisposition to thrombus formation and thromboembolic complications [7].

In our experience, we had concerns about the fate of the rings. Would we have to deal with a dislocation or a rupture of the neck? Was the polymer fairly moldable to achieve an effective reduction in ring volume? With these considerations, we decided to use a covered stent for protecting the aortic neck and maintaining long-term radial force. Although we are reporting an isolated experience, we believe that aortic stenting for severe neck narrowing following use of the Ovation Endograft is safe and feasible. IVUS technology facilitates proper delivery of the stent and permits evaluation of the final squeezing of the ring polymer.

The Ovation stent graft may help expand the patient population eligible for EVAR by accommodating a wider range of aortoiliac anatomies, especially a large neck. However, its advantages are offset by inflow stenosis and increased risk of thrombotic complications when the sizing of the endograft is suboptimal. Our experience shows that polymer rings are moldable, and a bail-out procedure is feasible when severe stenosis appears.

\section{Conflict of Interest}

The authors have no conflicts of interest relevant to this publication.

\section{Comment on this Article or Ask a Question}

\section{References}

1. Jackson RS, Chang DC, Freischlag JA. Comparison of long-term survival after open vs endovascular repair of intact abdominal aortic aneurysm among Medicare beneficiaries. JAMA. 2012;307:1621-1628. DOI: 10.1001/jama.2012.453

2. Greenhalgh RM, Brown LC, Powell JT, Thompson SG, Epstein D, Sculpher MJ. Endovascular versus open repair of abdominal aortic aneurysm. N Engl J Med. 2010;362:1863-1871. DOI: 10.1056/NEJMoa0909305

3. Harris PL, Buth J, Mialhe C, Myhre HO, Norgren $L$. The need for clinical trials of endovascular abdominal aortic aneurysm stent-graft repair: the EUROSTAR project. J Endovasc Surg. 1997;4:72e7. DOI: 10.1583/1074-6218(1997)004<0072:TNFCTO > 2.0.CO;2

4. Cuypers Ph, Buth J, Harris PL, Gevers E, Lahey R. On behalf of the EUROSTAR Collaborators. Reprinted Article "Realistic
Expectations for Patients with Stentgraft Treatment of Abdominal Aortic Aneurysms. Results of a European Multicentre Registry". Eur J Vasc Endovasc Surg. 2011;42:S63-S71. DOI: 10.1016/j. ejvs.2011.06.012

5. Mangialardi N, Ronchey $\mathrm{S}$, Kasemi H, Alberti V, Fazzini S, Serrao E. Percutaneous endovascular aneurysm repair with the ultra-low profile Ovation abdominal stentgraft system. J Cardiovasc Surg (Torino). 2013;54:581-587. PMID: 24002387

6. Mehta M, Valdes FE, Nolte T, Mishkel GJ, Jordan WD, Gray B, et al. One-year outcomes from an international study of the Ovation abdominal stent graft system for endovascular aneurysm repair. J Vasc Surg. 2014;59:65-73.e1-3. DOI: 10.1016/j. jvs.2013.06.065

7. Ioannou CV, Kontopodis N, Metaxa E, Papaharilaou Y, Georgakarakos E, Kafetzakis
A, et al. Graft inflow stenosis induced by the inflatable ring fixation mechanism of the Ovation stent-graft system: hemodynamic and clinical implications. J Endovasc Ther. 2014;21:829-838. DOI: 10.1583/14-4771MR.1

8. Aristokleous N, Kontopodis NG, Tzirakis K, Ioannou CV, Papaharilaou Y. Hemodynamic impact of abdominal aortic aneurysm stent-graft implantation-induced stenosis. Med Biol Eng Comput. 2016;54:1523-1532. DOI: 10.1007/s11517-015-1425-1

Cite this article as: Bianchi $P$, Scalise $F$, Mortara A, Lanzillo G, Scardina G, Trimarchi S, Parati G, Tolva V. Occlusive Shrinkage of Ovation Endograft Presenting as Acute Lower Limb Ischemia: Effective Endovascular Management. AORTA (Stamford). 2017;5(1):21-26. DOI: http:// dx.doi.org/10.12945/j.aorta.2017.16.041 